\title{
Cervical cancer update: The latest on screening \& management
}

\section{Here are updated guidelines for prevention, testing, and treatment. Elimination of causative HPV continues to hold center stage in the global effort to curb disease.}

\section{PRACTICE RECOMMENDATIONS \\ $>$ Encourage eligible patients to be vaccinated against human papillomavirus (HPV) because the vaccine is highly effective for preventing cervical dysplasia, especially when given to patients previously unexposed to the virus. (A)}

> Screen for cervical disease with either cytology plus HPV testing or primary HPV testing with secondary triage for cytology; both protocols are more accurate than screening with cervical cytology alone, and allow you to widen the screening interval. (A)

Strength of recommendation (SOR)

(A) Good-quality patient-oriented evidence

B Inconsistent or limited-quality patient-oriented evidence

(C) Consensus, usual practice, opinion, disease-oriented evidence, case series
7 he World Health Organization estimates that, in 2020, worldwide, there were 604,000 new cases of uterine cervical cancer and approximately 342,000 deaths, $84 \%$ of which occurred in developing countries. ${ }^{1}$ In the United States, as of 2018, the lifetime risk of death from cervical cancer was 2.2 for every 100,000 , with a mean age of 50 years at diagnosis. ${ }^{2}$

In this article, we summarize recent updates in the epidemiology, prevention, and treatment of cervical cancer. We emphasize recent information of value to family physicians, including updates in clinical guidelines and other pertinent national recommendations.

\section{Spotlight continues to shine on HPV}

It has been known for several decades that cervical cancer is caused by human papillomavirus (HPV). Of more than 100 known HPV types, 14 or 15 are classified as carcinogenic. HPV 16 is the most common oncogenic type, causing more than $60 \%$ of cases of cervical cancer ${ }^{3,4}$; HPV 18 is second, causing $\mathbf{1 6 . 5 \%}$ of cases-taken together, the 2 types account for more than $75 \%$ of cervical cancers.

HPV is the most common sexually transmitted infection, with as many as $80 \%$ of sexually active people becoming infected during their lifetime, generally before 50 years of age. ${ }^{5}$

\section{A NOTE FROM THE EDITORS}

The Editors of The Journal of Family Practice recognize the importance of addressing the reproductive health of gender-diverse individuals. In this article, we use the words "women," "men," "girls," and "boys" in limited circumstances (1) for ease of reading and (2) to reflect the official language of the US Food and Drug Administration and the Advisory Committee on Immunization Practices. The reader should consider the information and guidance offered in this discussion of cervical cancer and other human papillomavirus-related cancers to speak to the care of people with a uterine cervix and people with a penis.

\author{
Linda Speer, MD; \\ Shirley Bodi, MD \\ Department of Family \\ Medicine, University of \\ Toledo College of Medicine \\ and Life Sciences, $\mathrm{OH}$
}

D Linda.speer@utoledo.edu

The authors reported no potential conflict of interest relevant to this article.

doi: $10.12788 /$ jfp.0316 
TABLE 1

\section{Variables associated with cervical cancer ${ }^{7}$}

\begin{tabular}{l} 
Cigarette smokinga $^{\text {a }}$ \\
\hline Early onset of sexual activity \\
\hline Genetic predisposition to persistent human papillomavirus infection
\end{tabular}

History of other sexually transmitted infection

Immunosuppression

Multiple sexual partners or a high-risk sexual partner

${ }^{a}$ Associated with an increased risk of squamous cell carcinoma but not an increased risk of adenocarcinoma.
Gardasil 9 (9-valent vaccine, recombinant; Merck), which provides coverage for 7 highrisk HPV types that account for approximately $90 \%$ of cervical cancers and 2 types ( 6 and 11 ) that are the principal causes of condylomata acuminata (genital warts). Future generations of prophylactic vaccines are expected to cover additional strains.

Vaccine studies have been summarized in a Cochrane review, ${ }^{11}$ showing that vaccination is highly effective for prevention of cervical dysplasia, especially when given to young girls and women ${ }^{a}$ previously unexposed to the virus. It has not been fully established how long protection lasts, but vaccination appears to be $70 \%$ to $90 \%$ effective for $\geq 10$ years.

Dosing schedule. The Advisory Committee on Immunization Practices (ACIP) of the Centers for Disease Control and Prevention (CDC) recommends a 2-dose schedule 6 to 15 months apart, for both girls and boys between 9 and 14 years of age. ${ }^{12} \mathrm{~A}$ third dose is indicated if the first and second doses were given less than 5 months apart, or the person is older than 15 years or is immunocompromised. No recommendation has been made for revaccination after the primary series.

In 2018, the US Food and Drug Administration approved Gardasil 9 for adults 27 to 45 years of age. In June 2019, ACIP recommended vaccination for men as old as 26 years, and adopted a recommendation that unvaccinated men and women between 27 and 45 years discuss HPV vaccination with their physician..$^{13}$

The adolescent HPV vaccination rate varies by state; however, all states lag behind the CDC's Healthy People 2020 goal of $80 \% .{ }^{14}$ Barriers to vaccination include cost, infrastructure limitations, and social stigma.

\section{Secondary prevention: Screening and Tx of precancerous lesions}

Cervical cancer screening identifies patients at increased risk of cervical cancer and reassures the great majority of them that their risk of cervical cancer is very low. There are 3 general approaches to cervical cancer screening:

- cytology-based screening, which has been implemented for decades in many countries 
TABLE 2

\section{US Preventive Services Task Force recommendations for cervical cancer screening ${ }^{15}$}

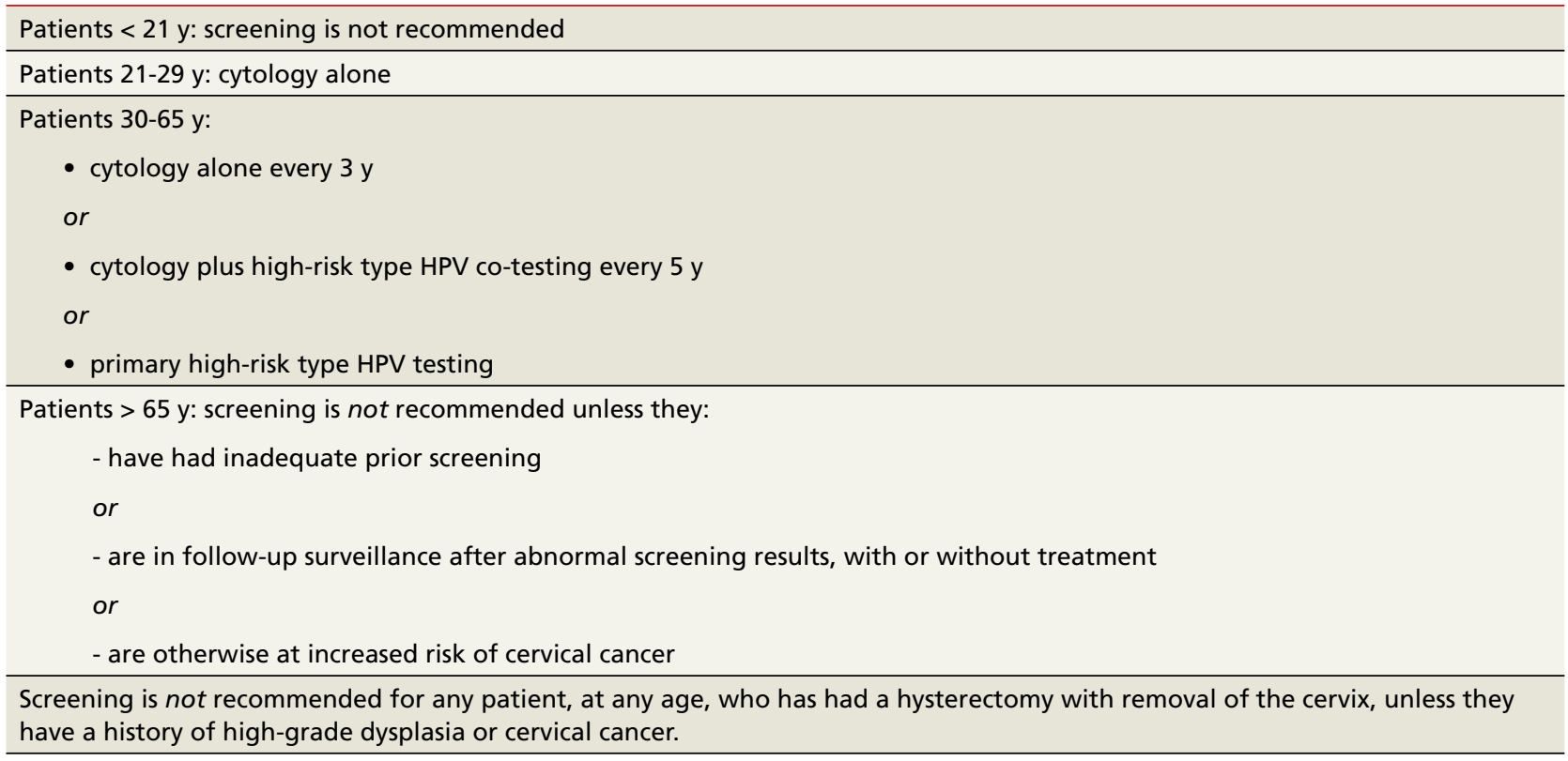

HPV, human papillomavirus.

- primary testing for DNA or RNA markers of high-risk HPV types

- co-testing with cytology-based screening plus HPV testing.

I USPSTF guidance. Recommendations of the US Preventive Services Task Force (USPSTF) for cervical cancer screening were updated in 2018 (TABLE $\mathbf{2}^{15}$ ). The recommendations state that high-risk HPV screening alone is a strategy that is amenable to patient self-sampling and self-mailing for processing-a protocol that has the potential to improve access to testing for patients who are inadequately screened.

I ASCCP guidance. The American Society of Colposcopy and Cervical Pathology (ASCCP) makes nearly the same recommendations for cervical cancer screening. An exception is that ASCCP guidelines allow for the possibility of screening using primary high-risk HPV testing for patients starting at 25 years of age. ${ }^{16}$

Screening programs that can be initiated at a later age and longer intervals should be possible once the adolescent vaccination rate is optimized and vaccination registries are widely implemented.

\section{Cervical cytology protocol}

Cervical cytologic abnormalities are reported using the Bethesda system. Specimen adequacy is the most important component of quality assurance, ${ }^{17}$ and is determined primarily by sufficient cellularity. However, any specimen containing abnormal squamous cells of undetermined significance (ASCUS) or atypical glandular cells (AGCs) is considered satisfactory, regardless of the number of cells. Obscuring factors that impair quality include excessive blood; inflammation; airdrying artifact; and an interfering substance, such as lubricant. The presence of reactive changes resulting from inflammation does not require further evaluation unless the patient is immunosuppressed.

Abnormalities are most often of squamous cells, of 2 categories: low-grade squamous intraepithelial lesions (LSILs) and high-grade squamous intraepithelial lesions (HSILs). HSILs are more likely to be associated with persistent HPV infection and higher risk of progression to cervical cancer.

Cytologic findings can be associated with histologic findings that are sometimes more, sometimes less, severe. LSIL cytology 


\section{$>$}

High-risk HPV screening alone is amenable to patient selfsampling and self-mailing for processing-a protocol that has the potential to improve access to testing. specimens that contain a few cells that are suspicious for HSIL, but that do not contain enough cells to be diagnostic, are reported as atypical squamous cells, and do not exclude a high-grade intraepithelial lesion.

Glandular-cell abnormalities usually originate from the glandular epithelium of the endocervix or the endometrium-most often, AGCs. Less frequent are AGCs, favor neoplasia; endocervical adenocarcinoma in situ; and ADC. Rarely, AGCs are associated with adenosquamous carcinoma. Endometrial polyps are a typical benign pathology that can be associated with AGCs.

In about $30 \%$ of cases, AGCs are associated with premalignant or malignant disease. ${ }^{18}$ The risk of malignancy in patients with AGCs increases with age, from $<2 \%$ among patients younger than 40 years to approximately $15 \%$ among those $>50$ years. ${ }^{19}$ Endometrial malignancy is more common than cervical malignancy among patients $>40$ years.

AGC cytology requires endocervical curettage, plus endometrial sampling for patients $\geq 35$ years. Patients with a history of AGCs are at higher risk of cervical cancer for as long as 15 years.

Cytology-based screening has limitations. Sensitivity is relatively low and dependent on the expertise of the cytologist, although regular repeat testing has been used to overcome this limitation. A substantial subset of results are reported as equivocal-ie, ASCUS.

\section{Primary HPV screening}

Primary HPV testing was approved by the US Food and Drug Administration in 2015 and recommended as an appropriate screening option by professional societies.

In contrast to cytology-based screening, HPV testing has high sensitivity ( $\geq 90 \%$ ); the population-based negative likelihood ratio is near zero. ${ }^{20}$ This degree of sensitivity allows for extended screening intervals. However, primary HPV testing lacks specificity for persistent infection and high-grade or invasive lesions, which approximately doubles the number of patients who screen positive. The potential for excess patients to be referred for colposcopy led to the need for secondary triage.

I Instituting secondary triage. Cytology is, currently, the primary method of second- ary triage, reducing the number of referrals for colposcopy by nearly one-half, compared to referrals for all high-risk HPV results, and with better overall accuracy over cytology with high-risk HPV triage. ${ }^{21}$ When cytology shows ASCUS, or worse, refer the patient for colposcopy; alternatively, if so-called reflex testing for HPV types 16 and 18 is available and positive, direct referral to colposcopy without cytology is also appropriate.

In the future, secondary triage for cytology is likely to be replaced with improved technologies, such as immunostaining of the specimen for biomarkers associated with cervical precancer or cancer, or for viral genome methylation testing. ${ }^{22}$

\section{Management of abnormal cervical cancer screening results}

Routine screening applies to asymptomatic patients who do not require surveillance because they have not had prior abnormal screening results. In 2020, ASCCP published risk-based management consensus guidelines that were developed for abnormal cervical cancer screening tests and for cancer precursors. ${ }^{16}$ Guiding principles, and screening situations in which the guidelines can be applied, are summarized in TABLE $3 .^{16}$

ASCCP guidelines provide a framework to incorporate new data and technologies without major revision. The web-based ASCCP resource can be obtained at no cost at $\mathrm{http} / /$ asccp.org; there is also a smartphone app resource (\$9.99).

Some noteworthy scenarios in ASCCP risk-based management are:

- For unsatisfactory cytology with a negative HPV test or no HPV test, repeat age-based screening in 2 to 4 months. (Note: A negative HPV test might reflect an inadequate specimen; do not interpret this result as a true negative.)

- An absent transformation zone (ie, between glandular and squamous cervical cells) with an otherwise adequate specimen should be interpreted as satisfactory for screening in patients 21 to 29 years of age. For those $\geq 30$ years and with no HPV testing in this circumstance, HPV testing is preferred; 
TABLE 3

ASCCP guiding principles for 2019 recommendations ${ }^{16}$

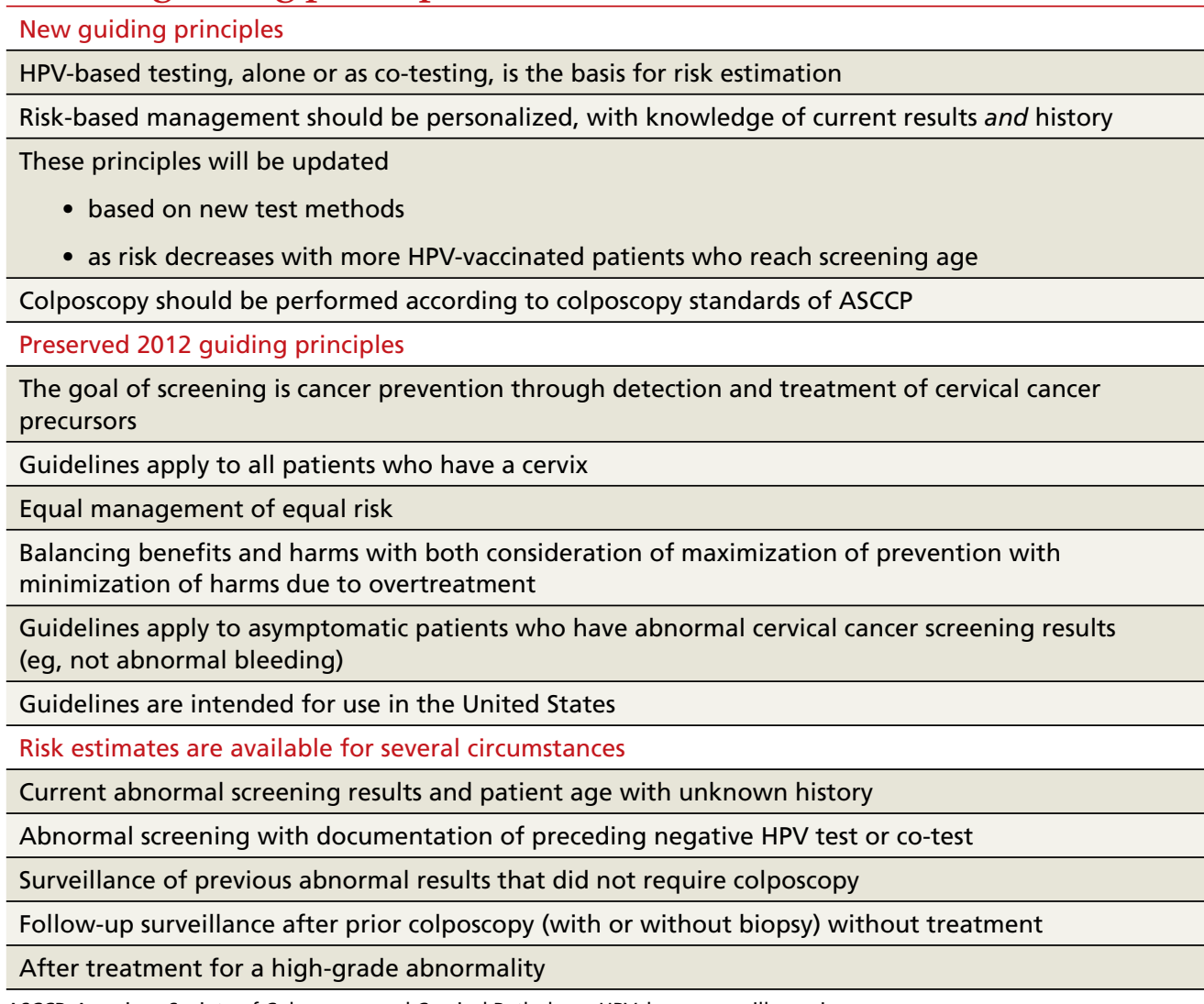

ASCCP, American Society of Colposcopy and Cervical Pathology; HPV, human papillomavirus.

repeating cytology, in 3 years, is also acceptable.

- After a finding of LSIL/CIN1 without evidence of a high-grade abnormality, and after 2 negative annual screenings (including HPV testing), a return to 3-year (not 5-year) screening is recommended.

- A cytology result of an HSIL carries a risk of $26 \%$ for $\mathrm{CIN} 3+$, in which case colposcopy is recommended, regardless of HPV test results.

- For long-term management after treatment for CIN2+, continue surveillance testing every 3 years after 3 consecutive negative HPV tests or cytology findings, for at least 25 years. If the 25-year threshold is reached before 65 years of age, continuing surveillance every 3 years is optional, as long as the patient is in good health (ie, life expectancy $\geq 10$ years).
- After hysterectomy for a high-grade abnormality, annual vaginal HPV testing is recommended until 3 negative tests are returned; after that, surveillance shifts to a 3-year interval until the 25-year threshold.

\section{Treatment of cancer precursors}

Treatment for cervical dysplasia is excisional or ablative.

Excisional therapy. In most cases, excisional therapy (either a loop electrosurgical excision procedure [LEEP; also known as large loop excision of the transformation zone, cold knife conization, and laser conization] or cone biopsy) is required, or preferred. Excisional treatment has the advantage of providing a diagnostic specimen.

The World Health Organization recommends LEEP over ablation in settings in which LEEP is available. ${ }^{23}$ ASCCP states that,
In about $30 \%$ of cases, atypical glandular cells (AGCs) found on cytology are associated with premalignant or malignant disease. The risk of malignancy with AGCs increases with age. 
TABLE 4

\section{Cervical lesions and other patient factors that require a diagnostic specimen ${ }^{16}$}

\begin{tabular}{l} 
Lesion extends into the cervical canal, where it cannot be fully visualized \\
Lesion covers $\geq 75 \%$ of the exocervix or extends beyond the reach of the \\
cryoablation tip, or both \\
\hline Endocervical curettage shows CIN2+ or ungraded CIN \\
\hline Patient had prior excision for CIN2+ \\
\hline Treatment is for glandular disease \\
Diagnostic uncertainty because of inadequate colposcopy or discordant \\
cytology and biopsy results
\end{tabular}

CIN, cervical intraepithelial neoplasia.

in the relatively few cases in which treatment is needed and it is for CIN1, either excision or ablation is acceptable. TABLE $4^{16}$ lists situations in which excisional treatment is required because a diagnostic specimen is needed.

Ablative treatments are cryotherapy, $\mathrm{CO}_{2}$ laser ablation, and thermal ablation. Ablative therapy has the advantage of presenting less risk of adverse obstetric outcomes (eg, preterm birth); it can be used if the indication for therapy is:

- CIN1 or CIN2 and HPV type 16 or 18 positivity

- concordant cytology and histology

- satisfactory colposcopy

- negative endocervical curettage.

The most common ablative treatment is liquid nitrogen applied to a metal tip under local anesthesia.

- Hysterectomy can be considered for patients with recurrent CIN2+ who have completed childbearing or for whom repeat excision is infeasible (eg, scarring or a short cervix), or both.

Cost, availability, and convenience might play a role in decision-making with regard to the treatment choice for cancer precursors.

Is care after treatment called for? $\mathrm{Pa}$ tients who continue to be at increased risk of (and thus mortality from) cervical and vaginal cancer require enhanced surveillance. The risk of cancer is more than triple for patients who were given their diagnosis, and treated, when they were $>60$ years, compared to patients treated in their 30s. ${ }^{1}$ The excess period of risk covers at least 25 years after treatment, even among patients who have had 3 posttreatment screenings.

Persistent HPV positivity is more challenging. Patients infected with HPV type 16 have an increased risk of residual disease.

\section{Cancer management}

Invasive cancer. Most cervical cancers (60\%) occur among patients who have not been screened during the 5 years before their diagnosis. ${ }^{24}$ For patients who have a diagnosis of cancer, those detected through screening have a much better prognosis than those identified by symptoms (mean cure rate, $92 \%$ and $66 \%$, respectively). ${ }^{25}$ The median 5 -year survival for patients who were not screened during the 5 years before their diagnosis of cervical cancer is $66 \%{ }^{2}$

In unscreened patients, cervical cancer usually manifests as abnormal vaginal bleeding, especially postcoitally. In approximately $45 \%$ of cases, the patient has localized disease at diagnosis; in 36\%, regional disease; and in $15 \%$, distant metastases. ${ }^{26}$

For cancers marked by stromal invasion $<3 \mathrm{~mm}$, appropriate treatment is cone biopsy or simple hysterectomy. ${ }^{27}$

Most patients with early-stage cervical cancer undergo modified radical hysterectomy. The ovaries are usually conserved, unless the cancer is adenocarcinoma. Sentinel-node dissection has become standard practice. Primary radiation therapy is most often used for patients who are a poor surgical candidate because of medical comorbidity or poor functional status. Antiangiogenic agents (eg, bevacizumab) can be used as adjuvant palliative therapy for advanced and recurrent disease. $^{28}$

After treatment for invasive cervical cancer, the goal is early detection of recurrence, although there is no consensus on a protocol. Most recurrences are detected within the first 2 years.

Long-term sequelae after treatment for advanced cancer are considerable. Patients report significantly lower quality of life, comparatively, across multiple dimensions, including mental health, physical health, and sexual function. ${ }^{29}$

Hormone replacement therapy is gener- 
ally considered acceptable after treatment of cervical cancer because it does not increase replication of HPV.

I Recurrent or metastatic cancer. Recurrence or metastases will develop in $15 \%$ to $60 \%$ of patients, ${ }^{30}$ usually within the first 2 years after treatment.

Management depends on location and extent of disease, using mainly radiation therapy or surgical resection. Recurrence or metastasis is usually incurable.

Last, there are promising areas of research for more effective treatment for cervical cancer precursors and cancers, including gene editing tools ${ }^{31}$ and therapeutic vaccination, ${ }^{32}$ which is intended to target and kill infected cells.

\section{Prospects for better cervical cancer care}

I Prevention. HPV vaccination is likely to have a large impact on population-based risk of both cancer and cancer precursors in the next generation.

I Screening in the foreseeable future will gravitate toward reliance on primary HPV screening, with a self-sampling option.

$\checkmark$ Surveillance after dysplastic disease. The 2019 ASCCP guidelines for surveillance and intervention decisions after abnormal cancer screening results will evolve to incorporate introduction of new technology into computerized algorithms.

I Treatment. New biologic therapies, including monoclonal antibodies and therapeutic vaccines against HPV, will likely be introduced for treating cancer precursors and invasive cancer.

\section{CORRESPONDENCE}

Linda Speer, MD, 3000 Arlington Avenue, MS 1179, Toledo, OH 43614; Linda.speer@utoledo.edu

\section{References}

1. Sung H, Ferlay J, Siegel RL, et al. Global cancer statistics 2020: GLOBOCAN estimates of incidence and mortality worldwide for 36 cancers in 185 countries. CA Cancer J Clin. 2021;71:209-249. doi: $10.3322 /$ caac. 21660

2. Cancer stat facts: cervical cancer. National Cancer Institute Surveillance, Epidemiology, and End Results [SEER] Program. Accessed November 14, 2021. https://seer.cancer.gov/statfacts/ html/cervix.html

3. Guan P, Howell-Jones R, Li N, et al. Human papillomavirus types in 115,789 HPV-positive women: a meta-analysis from cervical infection to cancer. Int J Cancer 2012;131:2349-2359. doi: 10.1002/ ijc. 27485
4. Winer RL, Hughes JP, Feng Q, et al. Early history of incident type-specific human papillomavirus infections in newly sexually active young women. Cancer Epidemiol Biomarkers Prev 2011;20:699-707. doi: 10.1158/1055-9965.EPI-10-1108

5. Chesson HW, Dunne EF, Hariri F, et al. The estimated lifetime probability of acquiring human papillomavirus in the United States. Sex Transm Dis. 2014;41:660-664. doi: 10.1097/ OLQ.0000000000000193

6. Human papillomavirus (HPV) and cervical cancer. Fact sheet. Geneva, Switzerland: World Health Organization; November 11 2020. Accessed November 14, 2021. www.who.int/news-room/factsheets/detail/human-papillomavirus-(hpv)-and-cervical-cancer

7. International Collaboration of Epidemiological Studies of Cervical Cancer. Comparison of risk factors for invasive squamous cell carcinoma and adenocarcinoma of the cervix: collaborative reanalysis of individual data on 8,097 women with squamous cell carcinoma and 1,374 women with adenocarcinoma from 12 epidemiological studies. Int J Cancer. 2007;120:885-891. doi: 10.1002/ijc.22357

8. McCredie MRE, Sharples KJ, Paul C, et al. Natural history of cervical cancer neoplasia and risk of invasive cancer in women with cervical intraepithelial neoplasia 3: a retrospective cohort study. Lancet Oncol. 2008:9:425-434. doi: 10.1016/ S1470-2045(08)70103-7

9. de Sanjose S, Quint WG, Alemany I, et al; Retrospective International Survey and HPV Time Trends Study Group. Human papillomavirus genotype attribution in invasive cervical cancer: a retrospective, cross-sectional worldwide study. Lancet Oncol. 2010;11:1048-1056. doi: 10.1016/S1470-2045(10)70230-8

10. Ries LAG, Melbert D, Krapcho M, et al. SEER Cancer Statistics Re view 1975-2004. Bethesda, MD: National Cancer Institute; 2007. Accessed November 14, 2021. https://seer.cancer.gov/archive/ csr/1975_2004/\#citation

11. Arbyn M, Xu L, Simoens C, et al. Prophylactic vaccination against human papillomaviruses to prevent cervical cancer and its precursors. Cochrane Database Syst Rev. 2018;5:CD009069. doi: 10.1002/14651858.CD009069.pub3

12. Meites E, Kempe A, Markowitz LE. Use of a 2-dose schedule for human papillomavirus vaccination - updated recommendations of the Advisory Committee on Immunization Practices. MMWR Morb Mortal Wkly Rep. 2016:65;1405-1408. doi: 10.15585/mmwr. mm6549a5

13. Meites E, Szilagyi PG, Chesson HW, et al. Human papillomavirus vaccination for adults: updated recommendations of the Advisory Committee on Immunization Practices. $M M W R$ Morb Mortal Wkly Rep. 2019;68:698-702. doi: 10.15585/mmwr mm6832a3

14. State-level data: Female adolescents receiving 2 or 3 doses of HPV vaccine by age 13-15 years (percent). HealthyPeople.gov. Accessed November 14, 2021. www.healthypeople.gov/2020/data/ map $/ 4657$ ? year $=2018$

15. United States Preventive Services Task Force; Curry SJ, Kris $\mathrm{AH}$, Owens DK, et al. Screening for cervical cancer: US Preventive Services Task Force recommendation statement. JAMA 2018;320:674-686. doi: 10.1001/jama.2018.10897

16. Perkins RB, Guido RS, Castle PE, et al; 2019 ASCCP Risk-Based Management Consensus Guidelines Committee. 2019 ASCCP risk-based management consensus guidelines for abnormal cervical cancer screening tests and cancer precursors. J Low Genit Tract Dis. 2020;24:102-131. doi: 10.1097/LGT.0000000000000525

17. Nayar R, Wilbur DC. The Pap test and Bethesda 2014. Cancer Cytopathol. 2015;123;271-281. doi: 10.1002/cncy.21521

18. Schnatz PF, Guile M, O'Sullivan DM, et al. Clinical significance of atypical glandular cells on cervical cytology. Obstet Gynecol 2006;107:701-708. doi: 10.1097/01.AOG.0000202401.29145.68

19. Zhao C, Florea A, Onisko A, et al. Histologic follow-up results in 662 patients with Pap test findings of atypical glandular cells: results from a large academic womens hospital laboratory employing sensitive screening methods. Gynecol Oncol 2009;114 383-389. doi: 10.1016/j.ygyno.2009.05.019

20. Zazove P, Reed BD, Gregoire L, et al. Low false-negative rate of PCR analysis for detecting human papillomavirus-related cervical lesions. J Clin Microbiol. 1998;36:2708-2713. doi: 10.1128/ JCM.36.9.2708-2713.1998

21. Richardson LA, El-Zein M, Ramankumar AV, et al; PEACHS (Pap Efficacy After Cervical HPV Status) Study Consortium. HPV DNA testing with cytology triage in cervical cancer screening: influence of revealing HPV infection status. Cancer Cytopathol. 2015:123:745-754. doi: 10.1002/cncy.21596

22. Wentzensen N, Schiffman M, Palmer T, et al. Triage of HPV CONTINUED ON PAGE 509
After treatment for invasive cervical cancer, the goal is early detection of recurrence. Most recurrences are detected within the first 2 years. 


\section{CONTINUED FROM PAGE 505}

positive women in cervical cancer screening. J Clin Virol 2016;76 S49-S55. doi: 10.1016/j.jcv.2015.11.015

23. WHO Guidelines: Use of Cryotherapy for Cervical Intraepithelial Neoplasia. Geneva, Switzerland: World Health Organization 2011. Accessed November 14, 2021. www.ncbi.nlm.nih.gov/ books/NBK138476/pdf/Bookshelf_NBK138476.pdf

24. Spence AR, Goggin P, Franco EL. Process of care failures in invasive cervical cancer: systematic review and meta-analysis. Prev Med. 2007:45:93-106. doi: 10.1016/j.ypmed.2007.06.007

25. Rositch AF, Nowak RG, Gravitt PE. Increased age and race-specific incidence of cervical cancer after correction for hysterectomy prevalence in the United States from 2000-2009. Cancer. 2014:120:2032-2038. doi: $10.1002 /$ cncr.28548

26. Siegel RL, Miller KD, Fuchs HE, et al. Cancer statistics, 2021. CA Cancer J Clin. 2021;71:7-33. doi: 10.3322/caac.21654

27. National Comprehensive Cancer Network. Clinical practice guidelines in oncology: cervical cancer. Accessed June 15, 2021. www.nccn.org/professionals/physician_gls/pdf/cervical.pdf
28. Tewari KS, Sill MW, Penson RT, et al. Bevacizumab for advanced cervical cancer: final overall survival and adverse event analysis of a randomised, controlled, open-label, phase 3 trial (Gynecologic Oncology Group 240). Lancet. 2017;390:1654-1663. doi: 10.1016/S0140-6736(17)31607-0

29. Osann K, Hsieh S, Nelson EL, et al. Factors associated with poor quality of life among cervical cancer survivors: implications for clinical care and clinical trials. Gynecol Oncol. 2014;135:266-272. doi: 10.1016/j.ygyno.2014.08.036

30. Ries LAG, Harkins D, Krapcho M, et al. SEER Cancer Statistics Review, 1975 to 2003. Bethesda, MD: National Cancer Institute; 2007. Accessed November 14, 2021. https://seer.cancer.gov/ archive/csr/1975_2003/\#citation

31. Hu Z, Ding M. The precision prevention and therapy of HPVrelated cervical cancer: new concepts and clinical implications Cancer Med. 2018;7:5217-5236. doi: 10.1002/cam4.1501

32. Wang R, Pan W, Jin L, et al. Human papillomavirus vaccine against cervical cancer: opportunity and challenge. Cancer Lett. 2020;471:88-102. doi: 10.1016/j.canlet.2019.11.039 